\title{
Komorbiditäten bei Patienten mit juveniler idiopathischer Arthritis
}

\author{
Daten aus dem deutschen BiKeR-Register
}

\section{Comorbidities in patients with juvenile idiopathic arthritis}

\author{
Data from the German BiKeR register
}

Autoren
Ramona Napp, Gerd Horneff
Institut
Asklepios Klinik Sankt Augustin
Schlüsselwörter
Juvenile idiopathische Arthritis, Biologika, Komorbidität

Keywords

Juvenile idiopathic arthritis, biologicals, comorbidity

Bibliografie

DOI https://doi.org/10.1055/a-1120-2310

arthritis + rheuma 2020; 40: 184-198

(c) Georg Thieme Verlag KG Stuttgart · New York

ISSN 0176-5167

Korrespondenzadresse

Prof. Dr. Gerd Horneff

Asklepios Klinik Sankt Augustin

Arnold-Janssen Str. 29, 53757 Sankt Augustin

g.horneff@asklepios.com

\section{ZUSAMMENFASSUNG}

Ziele Untersuchung von Komorbiditäten bei juveniler idiopathischer Arthritis und Einfluss von JIA-Kategorie, Geschlecht und Therapie.

Methodik Datenbank des BiKeR Registers (Biologika-in-derKinderrheumatologie-Register), einer prospektiven, multizentrischen nichtinterventionellen Beobachtungsstudie. Klassifikation von 51 Komorbiditäten nach MedDRA unter Verwendung der Low-Level-Terms in 15 übergeordnete Kategorien. Vorbestehende Komorbiditäten wurden zur Bestimmung der Prävalenz, auftretende für die Inzidenz verwendet. Zum Vergleich wurden Häufigkeit bei Kindern und Jugendlichen nach Literatur verwendet.

Ergebnisse Bei 898 von 3804 Patienten wurden 1141 prävalente und 658 inzidente Komorbiditäten während 12527,5 Beobachtungsjahren dokumentiert. $1 / 3$ aller Komorbiditäten waren ophthalmologische Erkrankungen, Uveitis und deren Komplikationen. Betroffen waren vor allem Patienten mit Oligoarthritis. In absteigender Reihung folgten Hashimoto-
Thyreoiditis, Makrophagenaktivierungssyndrom (MAS), Allergien, Asthma bronchiale, Psoriasis und Vaskulitis. Atopische Erkrankungen waren seltener als erwartet. Muskuloskelettale Erkrankungen wie M. Perthes, Osteochondritis dissecans, Osteoporose und Bandscheibenvorfall waren häufiger, eine Skoliose seltener. Malignome kamen signifikant häufiger vor, insbesondere bei sJIA. Patienten mit sJIA hatten auch signifikant häufiger ein MAS und eine arterielle Hypertonie. Eine Adipositas war bei JIA nicht häufiger, wohl aber ein Diabetes mellitus.

Diskussion Patienten mit JIA zeigen eine Vielzahl von Komorbiditäten. Einige Komorbiditäten verdienen wegen ihrer Relevanz für die Morbidität und Mortalität im Langzeitverlauf besondere Aufmerksamkeit, vor allem Uveitis, muskuloskelettale Erkrankungen, Diabetes mellitus als kardiovaskulärer Risikofaktor und Malignome.

\section{ABSTRACT}

Introduction Examination of comorbidities in juvenile idiopathic arthritis and influence of JIA category, gender and therapy.

Methods Database of the BiKeR Register (Biologics in-therheumatology register), a prospective, multicentre noninterventional observational study. Classification of $51 \mathrm{co}-$ morbidities according to MedDRA low-level terms into 15 superordinate categories. Existing comorbidities were used to determine prevalence, newly occurring comorbidities were used to calculate incidence. For comparison, literature data were used where available for children and adolescents.

Results In 898 of 3804 patients 1141 prevalent and 658 incidental comorbidities during 12527.5 years of observation were documented. About one third of all comorbidities were ophthalmological diseases, uveitis and their complications. Patients with oligoarthritis were particularly affected. In descending order Hashimoto thyroiditis followed and then Macrophage activation syndrome (MAS), allergies, bronchial asthma, psoriasis and vasculitis. Atopic diseases were less frequent than expected. Musculoskeletal diseases like Perthes disease, osteochondritis dissecans, osteoporosis and herniated discs were more frequent, a scoliosis was less common. Malignancies were 
significantly more common, especially with sJIA. Patients with sJIA also had significant more often a MAS and arterial hypertension. Obesity was not more common in JIA, but autoimmune diabetes mellitus.
Discussion Patients with JIA show a variety of comorbidities. Some comorbidities deserve to be treated because of their relevance for morbidity and mortality in the long-term special attention, especially uveitis, musculoskeletal diseases, diabetes mellitus as a cardiovascular risk factor and malignancies.
Bei der juvenilen idiopathischen Arthritis (JIA) besteht eine chronische Gelenkentzündung multifaktorieller Genese [1]. Die JIA wird seit 1997 in 7 Kategorien unterteilt nach Gelenkbefall und extraartikulären Manifestationen, die sich bezüglich Prognose, Krankheitsdauer, Outcome und Begleiterkrankungen unterscheiden. Die JIA-Kategorien unterscheiden sich nicht nur in der langfristigen Funktionseinschränkung [2], sondern auch in den vorliegenden Komorbiditäten [3]. Komorbiditäten haben einen entscheidenden Einfluss auf den im Erwachsenenalter erreichten Gesundheitszustand [3].

Die Literatur zu Komorbiditäten bei der JIA ist limitiert. Das deutsche Biologika-in-der-Kinderrheumatologie-Register (BiKeR), in das seit 2001 mehr als 4000 Patienten eingeschlossen wurden, bietet die Möglichkeit der Untersuchung von Komorbiditäten in einem großen Kollektiv von JIA-Patienten. Bereits bestehende Komorbiditäten können als prävalente Komorbiditäten und im Beobachtungszeitraum neu aufgetretene Komorbiditäten als inzidente Ereignisse untersucht werden. Die diagnostische Sicherheit der Diagnose JIA erscheint hoch, weil in diesem Register Daten zu Patienten gesammelt werden, die eine Basistherapie oder eine Therapie mit Biologika erhalten.

\section{Material und Methoden}

\section{Patienten}

Das BiKeR-Register ist eine laufende prospektive, multizentrische nichtinterventionelle Beobachtungsstudie, an der über 100 Einrichtungen in Deutschland und Österreich beteiligt sind. Seit dem 01.01.2001 werden Daten zur Wirksamkeit und Verträglichkeit einer Therapie mit Biologika an über 4000 Patienten erhoben und ebenso an einer Kontrollkohorte von über 1500 Patienten ohne eine Biologika-Therapie. Dokumentiert werden demografische Daten, Diagnosen, bisherige und aktuelle medikamentöse Therapie, Krankheitsaktivität, relevante Laborparameter, der Funktionsstatus mit dem Childhood Health Assessment Questionaire (CHAQ) und unerwünschte Ereignisse erfasst. Die aktuelle Untersuchung bezieht sich auf bis September 2016 erhobene Daten.

\section{Definition der Komorbiditäten}

Folgende Erkrankungen wurden in der Datenbank gezielt gesucht: gastrointestinale (Zöliakie, Morbus Crohn, Colitis ulcerosa, unklassifizierte chronisch entzündliche Darmerkrankungen), muskuloskelettale (Hüftdysplasie, Morbus Perthes, Epiphyseolysis capitis femoris, Osteochondritis dissecans, Skoliose, Spondylolisthesis, Bandscheibenvorfall, Osteoporose), Schmerzsyndrome, psychiatrische (Angststörung, Depression, Aufmerksamkeitsstörung), neurologische (Krampfanfälle/Epilepsie, Migräne), metabolische (Dia- betes mellitus Typ I und II, Adipositas, iatrogenes Cushing-Syndrom, Hyperlipidämie, immunologische (IgA-Mangel, Hypogammaglobulinämie), organbezogene Autoimmunerkrankungen (Hashimoto-Thyreoiditis, Nephritis, Multiple Sklerose), rheumatologische Autoimmunerkrankungen (Vaskulitis, Kollagenosen), atopische (Asthma, Allergie), Makrophagenaktivierungssyndrom, hämatologische (Anämie, Leukozytopenie), genetisch bedingte (Thalassämie, familiäres Mittelmeerfieber, Turner-Syndrom, Trisomie 21/ Down-Syndrom, Mikrodeletionssyndrom 22q), dermatologische (Alopezie, atopische Dermatitis, Raynaud-Syndrom, Urtikaria, Vitiligo, Psoriasis), kardiovaskuläre (strukturelle Herzfehler, arterielle Hypertonie), onkologische (Malignome, Leukämien), urologische (vesikourethraler Reflux), ophthalmologische (Myopie, Uveitis, Katarakt, Glaukom), otorhinologische Erkrankungen (Hörstörung, Schwindel, Nystagmus), Entwicklungsverzögerung und Kleinwuchs. Diabetes mellitus Typ I wurde gemeinsam mit Diabetes Typ II zu den metabolischen Erkrankungen gezählt. Im Case report form (CRF) wurde nicht zwischen Typ I und Typ II differenziert, jedoch tritt bei Kindern und Jugendlichen nur selten ein Diabetes mellitus Typ II auf. Sofern nicht eine angeborene/konnatale Hypothyreose bestand, wurde „Hypothyreose“ zur Hashimoto-Thyreoiditis gezählt. Die atopische Dermatitis und die Urtikaria wurden zu den dermatologischen Erkrankungen gezählt im Gegensatz zum Asthma bronchiale als organspezifische Immunerkrankung. Für die Körperhöhe wurden der Standard Deviation Score für das Alter und das Geschlecht berechnet. Kleinwüchsigkeit wurde als SDS $\leq-2$ definiert [4]. Der Body-Mass-Index (BMI) wurde aus der Körperhöhe und dem Gewicht und altersbezogen als Standard Deviation Score (SDS) berechnet [4]. Adipositas wurde als BMI-SDS $\geq 2$ definiert, was der 97,5\%igen Perzentile entspricht.

Alle bekannten Diagnosen sowie alle im Verlauf aufgetretenen unerwünschten Ereignisse werden nach MedDRA (Medical Dictionary for Regulatory Activities) kodiert. Die Prävalenz wird in Ereignisse pro 100000 Personen, die Inzidenz in Ereignisse pro 100000 Jahre angegeben.

\section{Ergebnisse}

Untersucht wurden 3804 JIA-Patienten, 1144 (30\%) mit Rheumafaktor(RF)-negativer Polyarthritis, 227 (6\%) mit RF-positiver Polyarthritis, 253 (7\%) mit systemischer Arthritis, 492 (13\%) mit persistierender Oligoarthritis, 678 (18\%) mit erweiterter Oligoarthritis (erwOA), 599 (16\%) mit Enthesitis-assoziierter Arthritis (ERA), 287 (8\%) mit Psoriasis-Arthritis (PsA) und 123 (3\%) mit unklassifizierter Arthritis, $67 \%$ der Patienten waren weiblich. Die mittlere Krankheitsdauer bei Registereinschluss war 3,3 Jahre, die Gesamtbeobachtung 12527,5 Patientenjahre, die mittlere Beobachtungsdauer war 3,3 Jahre. 
>Tab. 1 Anzahl prävalente Komorbiditäten/Patient.

\begin{tabular}{|c|c|c|c|c|c|}
\hline \multirow[b]{2}{*}{ JIA-Kategorie } & \multicolumn{3}{|c|}{ Prävalente Komorbiditäten } & \multicolumn{2}{|c|}{ Inzidente Komorbiditäten } \\
\hline & $\begin{array}{l}\text { Pat. mit Komorbi- } \\
\text { dität }\end{array}$ & $\begin{array}{l}\text { Anzahl Komorbidi- } \\
\text { täten }\end{array}$ & Rate/Patient & $\begin{array}{l}\text { Anzahl Komorbidi- } \\
\text { täten }\end{array}$ & Rate/Patient \\
\hline sJIA $(n=253)$ & $79(31 \%)$ & 116 & 0,46 & 72 & 0,28 \\
\hline RF--PA $(n=1144)$ & $246(22 \%)$ & 305 & 0,27 & 197 & 0,17 \\
\hline $\mathrm{RF}^{+}-\mathrm{PA}(\mathrm{n}=227)$ & $47(21 \%)$ & 58 & 0,26 & 31 & 0,14 \\
\hline persOA $(n=492)$ & $115(23 \%)$ & 141 & 0,29 & 68 & 0,14 \\
\hline erwOA $(n=678)$ & $181(27 \%)$ & 225 & 0,33 & 130 & 0,19 \\
\hline ERA $(n=599)$ & $144(24 \%$ & 195 & 0,33 & 89 & 0,15 \\
\hline PsA $(n=287)$ & $86(30 \%$ & 127 & 0,44 & 53 & 0,18 \\
\hline uJIA $(n=123)$ & $36(29 \%)$ & 49 & 0,40 & 18 & 0,15 \\
\hline
\end{tabular}

\section{Ophthalmologische Erk.}

Endokrinologische Erk.

Immunologische Erk.

Stoffwechselerk.

Dermatologische Erk.

Muskuloskelettale Erk.

Kardiologische Erk.

Psychiatrische Erk.

Hämatologische Erk.

Genetische Erk.

Gastrointestinale Erk.

Neurologische Erk.

Otorhinologische Erk.

Urologische Erk.

Onkologische Erk.

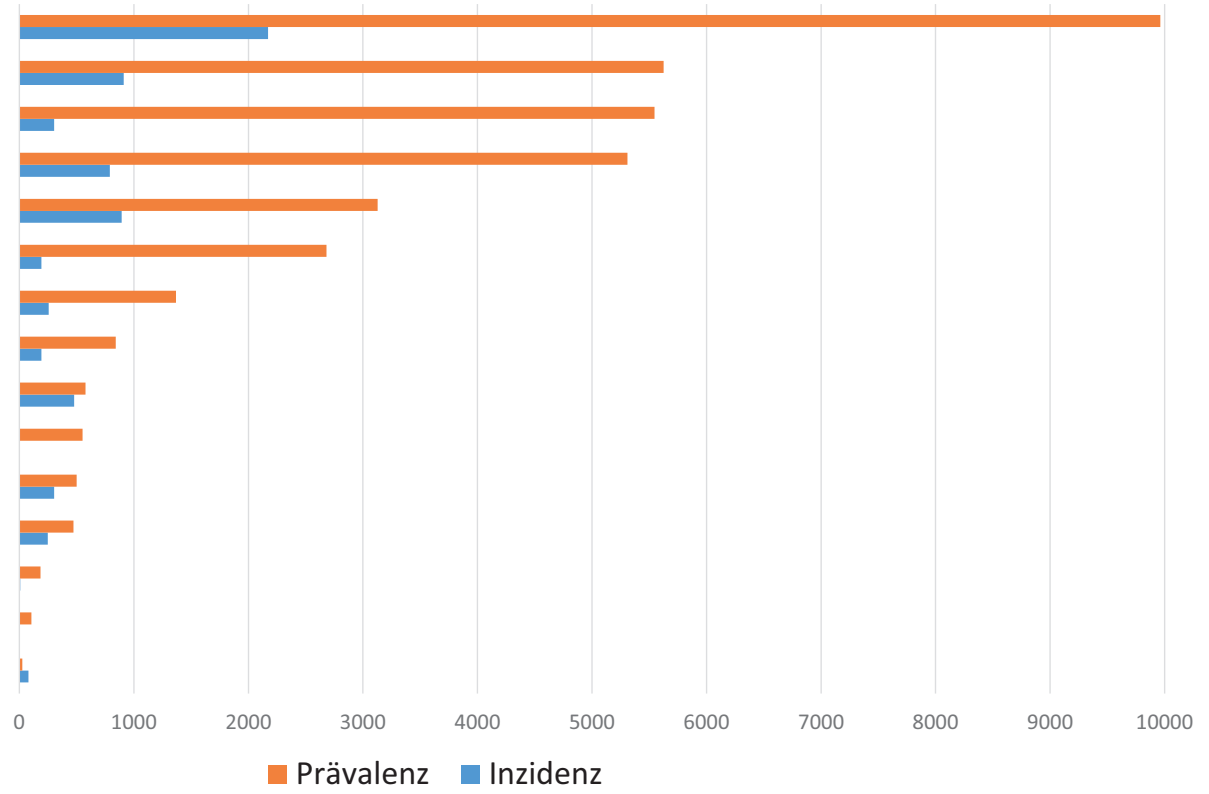

- Abb. 1 Prävalenz und Inzidenz von Komorbiditäten; Erk. = Erkrankungen.

\section{Komorbiditäten}

Bei Einschluss bestanden bereits zahlreiche Begleiterkrankungen, insbesondere Uveitis, Kleinwuchs, Adipositas und immunologische Komorbiditäten. Bei den inzidenten Komorbiditäten führen ebenso ophthalmologische, endokrinologische, immunologische und metabolische Komorbiditäten. Genetische Erkrankungen wurden erwartungsgemäß nur bei den prävalenten Komorbiditäten dokumentiert.

Insgesamt waren 1405 Komorbiditäten prävalent. Bei 1088 der 3804 Patienten (28,6\%) bestand zu Beginn der Beobachtung mindestens 1 Komorbidität, maximal 5 pro Patient (2 Patienten; 0,05\%), 6 Patienten (0,2\%) hatten 4 Komorbiditäten, 58 (1,5\%) 3, $188(4,9 \%) 2$ und 821 Patienten (21,6\%) 1 Komorbidität. Bei erwOA, PsA und unklassifizierter JIA (uJIA) bestanden mehr Komorbiditäten als bei der RF-negativen oder der RF-positiven Polyarthri- tis. Im Verlauf wurden 658 neue Komorbiditäten dokumentiert. In 420 Fällen bestand zuvor keine Komorbidität. Insgesamt traten bei Patienten mit sJIA am häufigsten Komorbiditäten hinzu ( $\triangleright$ Tab. 1).

\section{Häufigkeit der Komorbiditäten}

Sowohl bei den prävalenten wie auch bei den inzidenten Komorbiditäten waren ophthalmologische Erkrankungen am häufigsten ( $\vee$ Abb. 1). Diese machen $1 / 4$ der prävalenten und der inzidenten Komorbiditäten aus. In absteigender Reihenfolge folgen Wachstumsstörungen und Reifungsverzögerungen, metabolische Erkrankungen, immunologische, dermatologische und muskuloskelettale Komorbiditäten. Genetische Erkrankungen und angeborene Anomalien (z. B. Hüftdysplasie) wurden wie erwartet bei Ersterhebung und nicht mehr im Verlauf dokumentiert ( $\triangleright$ Tab. 2). Die gruppierten Komorbiditäten werden nachfolgend in der Reihenfolge absteigender Häufigkeiten detailliert beschrieben. 
- Tab. 2 Prävalenz und Inzidenz der Komorbiditäten.

\section{Komorbidität}

\section{Gastrointestinale Erkrankungen}

\section{Zöliakie}

Chronisch entzündliche Darmerkrankungen (M. Crohn, Colitis ulcerosa, unklassifizierte CED)

Fruktosmalabsorption

Laktoseintoleranz

Muskuloskelettale Erkrankungen

Hüftdysplasie

Morbus Perthes

Osteochondritis dissecans

Skoliose

Bandscheibenvorfall

Osteoporose

Schmerzsyndrome

Chronische nicht bakterielle Osteitis

Psychiatrische Erkrankungen

\begin{tabular}{|l|l|}
\hline Angststörung & 2 \\
\hline Depression & 6 \\
\hline Aufmerksamkeitsstörung & 24 \\
\hline Neurologische Erkrankungen & 18 \\
\hline Krampfanfälle/Epilepsie & 0 \\
\hline Migräne & 15 \\
\hline Entwicklungsverzögerung & \\
\hline Stoffwechselerkrankungen &
\end{tabular}

Diabetes mellitus (Typ I, Typ II)

Adipositas

Cushingoid (iatrogenes Cushing-Syndrom)

Immunologische Erkrankungen

IgA-Mangel
Hypogammaglobulinämie
Hashimoto-Thyreoiditis
Makrophagenaktivierungssyndrom
Allergie
Asthma
Vaskulitis

\section{Hämatologische Erkrankungen}

Anämie

Leukozytopenie

21

2

158

24

$18 \quad 473$

0

15

\section{1}

164

17

16

4

51

10

61

65

4

Genetische Erkrankungen

Thalassämie

Familiäres Mittelmeerfieber

Turner-Syndrom

Trisomie 21 (Down-Syndrom)

\section{Dermatologische Erkrankungen}

Alopezie

Atopische Dermatitis

7

52

3

9

2
Prävalenz Fälle/100 000 Neu beobachtet (n)

Inzidenz Fälle/100 000 Jahre

\begin{tabular}{|l|l|l|}
\hline 131 & 2 & 16 \\
\hline 0 & 32 & 152 \\
\hline 105 & 2 & 16 \\
\hline 263 & 2 & 16 \\
\hline
\end{tabular}

\begin{tabular}{|l|l|l|}
\hline 421 & 1 & 8 \\
\hline 158 & 1 & 8 \\
\hline 184 & 4 & 32 \\
\hline 552 & 1 & 8 \\
\hline 105 & 1 & 8 \\
\hline 315 & 2 & 6 \\
\hline 789 & 13 & 104 \\
\hline 158 & 1 & 8 \\
\hline
\end{tabular}

\begin{tabular}{l|l|l}
53 & 5 & 40
\end{tabular}

\begin{tabular}{l|l|l}
158 & 18 & 144
\end{tabular}

\begin{tabular}{l|l|l}
631 & 1 & 8
\end{tabular}

\begin{tabular}{l|l|l}
473 & 7 & 56
\end{tabular}

\begin{tabular}{l|l|l}
0 & 24 & 192
\end{tabular}

\begin{tabular}{l|l|l}
349 & 0 & 0
\end{tabular}

\begin{tabular}{|l|l|l|}
\hline 552 & 3 & 24 \\
\hline 4311 & 91 & 728 \\
\hline 447 & 5 & 40
\end{tabular}

\begin{tabular}{|l|l|l|}
\hline 421 & 1 & 8 \\
\hline 105 & 1 & 8 \\
\hline 1340 & 0 & 0 \\
\hline 263 & 12 & 96 \\
\hline 1604 & 19 & 152 \\
\hline 1709 & 2 & 16 \\
\hline 105 & 3 & 24 \\
\hline
\end{tabular}

\begin{tabular}{|l|l|l|}
552 & 2 & 16 \\
\hline
\end{tabular}

26

58

463

162


- Tab. 2 Fortsetzung

\begin{tabular}{|c|c|c|c|c|}
\hline Komorbidität & Vorbestehend (n) & Prävalenz Fälle/100000 & Neu beobachtet (n) & Inzidenz Fälle/100 000 Jahre \\
\hline Raynaud-Syndrom & 6 & 158 & 1 & 8 \\
\hline Urtikaria & 4 & 105 & 20 & 160 \\
\hline Psoriasis & 42 & 1104 & 12 & 96 \\
\hline Vitiligo & 8 & 210 & 0 & 0 \\
\hline \multicolumn{5}{|l|}{ Kardiologische Erkrankungen } \\
\hline Strukturelle Herzerkrankungen & 9 & 237 & 0 & 0 \\
\hline Funktionelle Herzerkrankungen & 17 & 447 & 10 & 80 \\
\hline Long QT-Syndrom & 1 & 26 & 12 & 96 \\
\hline Arterielle Hypertonie & 25 & 657 & 10 & 80 \\
\hline \multicolumn{5}{|l|}{ Onkologische Erkrankungen } \\
\hline Maligne Neoplasien & 1 & 26 & 10 & 80 \\
\hline \multicolumn{5}{|l|}{ Urologische Erkrankungen } \\
\hline Vesikoureteraler Reflux & 4 & 105 & 0 & 0 \\
\hline \multicolumn{5}{|c|}{ Ophthalmologische Erkrankungen } \\
\hline Myopie & 4 & 105 & 0 & 0 \\
\hline Uveitis & 360 & 9464 & 161 & 1285 \\
\hline Komplikationen der Uveitis & 15 & 394 & 11 & 88 \\
\hline \multicolumn{5}{|l|}{ Otorhinologische Erkrankungen } \\
\hline Hörstörung & 7 & 184 & 1 & 8 \\
\hline \multicolumn{5}{|l|}{ Endokrinologische Erkrankungen } \\
\hline Kleinwuchs & 199 & 5231 & 114 & 912 \\
\hline
\end{tabular}

- Tab. 3 JIA-Kategorien: Häufigkeit der Uveitis.

\begin{tabular}{|l|l|l|l|}
\hline & Uveitisfälle & Odd's Ratio [95\% KI] ${ }^{*}$ & P \\
\hline SJIA & $2(0,8 \%)$ & $0,07[0,02-0,28]$ & $<0,001$ \\
\hline RF--PA & $66(5,8 \%)$ & $0,5[0,4-0,7]$ & $<0,001$ \\
\hline RF'-PA & $3(1,3 \%)$ & $0,12[0,04-0,38]$ & $<0,001$ \\
\hline persOA & $85(17,3 \%)$ & $2,3[1,8-3,0]$ & $<0,001$ \\
\hline erwOA & $141(20,8 \%)$ & $3,5[2,8-4,4]$ & $<0,001$ \\
\hline ERA & $38(6,3 \%)$ & $0,6[0,4-0,9]$ & 0,004 \\
\hline PSA & $15(5,2 \%)$ & $0,5[0,3-0,9]$ & 0,01 \\
\hline UJIA & $10(8,1 \%)$ & $0,8[0,4-1,6]$ & 0,6 \\
\hline *Der Vergleich erfolgte mit den jeweils anderen JIA-Kategorien subsummiert.
\end{tabular}

Ophthalmologische Erkrankungen

Die Uveitis ist die häufigste Komorbidität der JIA mit einer Prävalenz von 9464/100 000 Patienten $(n=360)$. Patienten mit Oligoarthritis erkrankten signifikant häufiger, Patienten mit sJIA oder $\mathrm{RF}^{+}$-PA seltener ( $\vee$ Tab. 3 ). Signifikante Unterschiede in der Ge- schlechterverteilung gab es nicht. Die Inzidenz neu aufgetretener Uveitis war 1285/100000 ( $n=161)$. Patienten mit persistieren$\operatorname{der} O A$ (persOA) $(O R=1,6[95 \%-K I 1,1-2,5] ; p=0,02)$ oder erwOA $(O R=2,4[1,7-3,4] ; p<0,00001)$ waren signifikant häufiger, $\mathrm{Pa}$ tienten mit sJIA $(O R=0,08[0,01-0,6] ; p=0,00008)$ oder $R^{+}-P A$ 
$(O R=0,09[0,01-0,6] ; p=0,0002)$ seltener betroffen. Bei 15 Kindern wurde bei Ersterhebung mindestens 1 Komplikation der Uveitis berichtet (Prävalenz 394/100 000). Während der Beobachtung wurden 11 weitere Fälle mit Komplikation der Uveitis bekannt (Inzidenz 88/100 000).

\section{Endokrinologische Erkrankungen}

Kleinwuchs: Die Prävalenz (Längen SDS $\leq-2$ SDS) war $5231 / 100000$ Patienten ( $n=199)$. Jungen waren signifikant häufiger betroffen (OR 1,43[1,11-2,0]; $p=0,02$ ) ebenso Patienten mit sJIA (42/253; OR 4,4[3,1-6,4]; $p<0,001)$. Patienten mit persOA (9/492; OR =0,3 [0,2-0,6]; $p=0,0004)$ oder ERA (21/599 OR $0,6[0,4 ; 1] ; p=0,05)$ waren signifikant seltener kleinwüchsig. Zum Zeitpunkt der letzten Vorstellung zeigte sich ein Aufholwachstum der Patienten. $36 \%$ hatten sich im Verhältnis zu ihrem initialen SDS verbessert, $13 \%$ um mindestens 0,5 SDS, $5 \%$ um mindestens 1 SDS. Im Mittel waren bei Ersterhebung kleinwüchsige Kinder zum Zeitpunkt der letzten Erhebung 0,5 SDS größer.

\section{Immunologische Erkrankungen}

Die Prävalenz für IgA-Mangel war mit 421/100000 ( $n=16)$ höher als in der pädiatrischen Bevölkerung mit 1:500-1:3000 [5]. Bei erwOA war ein IgA-Mangel signifikant häufiger (7/378; OR 3,5 [1,39,7]; $p=0,007)$. Ein neuer Fall wurde berichtet (Inzidenz 8/100000).

Die Prävalenz der Hypogammaglobulinämie war $105 / 100000$ Patienten $(n=5)$. Ein weiterer Patient wurde im Beobachtungszeitraum bekannt (Inzidenz 8/100 000).

Für die Hashimoto-Thyreoiditis fand sich eine Prävalenz von $1340 / 100000(n=51)$ bei $1,6 \%$ in der allgemeinen pädiatrischen Bevölkerung [6]. Patienten mit PSA (12/287; OR 3,9[2,0-7,5]; $p<0,001), R^{+}-P A(8 / 227$; OR 2,9[1,4-6,3]; $p=0,003)$ und Mädchen (43/2548; OR 2,7[2,0-7,5]; $p=0,009)$ waren signifikant häufiger betroffen. Es wurde kein neuer Fall dokumentiert.

Ein Makrophagenaktivierungssyndrom bestand mit einer Prävalenz von 263/100 $000(n=10)$, besonders bei sJIA (9/253; OR 130[16-1037]; $p<0,0001$ ). Nur 1 Patient einer anderen JIA-Kategorie war betroffen. Im Beobachtungszeitraum zeigte sich eine Inzidenz von 96/100000 ( $n=12)$. Alle Patienten hatten eine sJIA. Ein MAS tritt bei 7-10\% der s]IA-Patienten auf [7, 8] und wurde mit einer Rate von 4,7\% nicht häufiger als erwartet beobachtet.

Mit einer Prävalenz von 1604/100 $000(n=61)$ wurden atopische Erkrankungen seltener dokumentiert als erwartet (10,5\%) [5]. ERA-Patienten waren signifikant häufiger betroffen als andere JIA-Kategorien (19/599; OR 2,4[1,4-4,2]; p =0,001). Die Inzidenz war mit 152/100 000 ebenso geringer.

Die Prävalenz von Asthma bei Kindern und Jugendlichen wird mit 7,2\% [5] angegeben. In BIKER war die Prävalenz 1709/100 000 Patienten $(n=65)$. Asthma war bei Jungen (31/1256; OR 1,9[1,2$3,1] ; p=0,01)$ und Patienten mit ERA (OR 2,0 [1,2-3,6]; $p=0,009)$ und PSA (OR 2,5[1,3-4,8]; $p=0,001$ ) häufiger. Die Inzidenz war $16 / 100000(n=2)$. Asthma wird bei JIA demnach seltener beobachtet als erwartet.

Zur Prävalenz einer Vaskulitis bei Kindern und Jugendlichen gibt es keine verlässlichen Daten. In BiKeR fand sich eine Prävalenz von $105 / 100000(n=4)$, und Inzidenz von 24/100 $000(n=3)$.

\section{Metabolische Erkrankungen}

Die Prävalenz für Diabetes mellitus Typ I in der pädiatrischen Bevölkerung ist 1400-2800/100 000 [6]. Bei 21 Kindern und Jugendlichen lag ein Diabetes vor (Prävalenz 552/100 000). Die Inzidenz war 24/100 000 bei 3 Neuerkrankungen.

Die Prävalenz der Adipositas (BMI-SDS $\geq 2$ ) war 4311/100 000. $1,1 \%$ der Kinder (42 Kinder) waren sehr adipös (SDS $\geq 3$ ). Signifikante Unterschiede zwischen den JIA-Kategorien oder den Geschlechtern bestanden nicht. In Deutschland sind 6,3\% der 2- bis 17-jährigen Kinder adipös, 15\% übergewichtig [9]. Der Vergleich des BMISDS bei Ersterhebung und letzter Dokumentation ergab, dass der Anteil adipöser Patienten von 4,3\% auf $5 \%$ anstieg. Es wurden weitere 26 Kinder adipös (Inzidenz 208/100 000). Auch der Anteil stark adipöser Patienten (SDS $\geq 3$ ) nahm um 0,4\% auf 1,5\% zu. $33 \%$ der Kinder, die schon zum Zeitpunkt der Ersterhebung adipös waren, hatten weiter zugenommen, $12 \%$ um mindestens 1 SDS. $9 \%$ hielten, $35 \%$ reduzierten ihren BMI um mindestens 1 SDS. Insgesamt zeigten JIA-Patienten keine höhere Adipositasrate.

Ein mutmaßlich medikamentös induziertes Cushingoid wurde bei 17 Patienten dokumentiert (Prävalenz 447/100 000). Vor allem Patienten mit sJIA waren betroffen (13/253; OR 48,0[15,5-148,4]; $\mathrm{p}<0,0001)$.

\section{Dermatologische Erkrankungen}

Die Prävalenz einer Alopezie war 184/100 000 ( $n=7)$, die Inzidenz 4150/100 000 Jahre $(n=52)$ ohne signifikante Unterschiede zwischen den Geschlechtern. Betroffen waren insbesondere sJIA-Patienten (7/252; $O R=2,2[1,0-5,0] ; p=0,05)$. Referenzwerte für Kinder und Jugendliche fanden sich nicht.

Die atopische Dermatitis zeigte eine Prävalenz von 1367/100 000 $(n=52)$, geringer als in der pädiatrischen Bevölkerung mit 5,6\% [6]. Die Inzidenz war 216/100 000 ( $n=27)$. Numerisch war eine atopische Dermatitis häufiger bei sJIA $(4 / 253$; OR = 2,4[0,8-7,1]; $\mathrm{p}=0,09)$. Bei $2 / 7$ mit Golimumab behandelten Patienten wurde eine inzidente atopische Dermatitis beobachtet $(O R=66[13-334]$; $\mathrm{p}=0,0007$ ).

Für ein Raynaud-Syndrom fand sich eine Prävalenz von 158/100 $000(n=6)$ ohne Präferenz einer JIA-Kategorie. Verlässliche Werte zum Auftreten eines Raynaud-Syndroms bei Kindern und Jugendlichen fanden sich nicht.

Die Prävalenz einer Urtikaria war 105/100 $000(n=4)$, die Inzidenz 160/100 $000(n=18)$. Belastbare Vergleichswerte fanden sich nicht, jedoch liegt die Lebenszeitprävalenz für Urtikaria bei $20 \%$ [10]. Betroffen waren 2/253 s]IA-Patienten (OR = 14[2-101]; $\mathrm{p}=0,02)$.

Die Prävalenz der Vitiligo war 210/100 000 Patienten ( $n=8)$. Bezüglich der zu erwartenden Prävalenz fanden sich sehr unterschiedliche Daten zwischen 0,06 bis 2,28\% [11].

Die Prävalenz der Psoriasis war 1104/100 000 Jahre $(n=42)$ im Vergleich zu 0,73 bei unter 20-Jährigen in Deutschland [12]. Patienten mit PsA waren erwartungsgemäß häufiger erkrankt ( $n=30$; $\mathrm{OR}=34$ [95\%-KI: (17-67)]; $\mathrm{p}=0$ ). Bei anderen JIA-Kategorien stellt die Psoriasis eine Ausschlussdiagnose dar. Die Inzidenz war 96/100000. 


\section{Muskuloskelettale Erkrankungen}

Eine Hüftdysplasie bestand bei 16 Kindern (Prävalenz 421/100 000). Im Verlauf ergab sich in einem Fall eine Neudiagnose einer Hüftdyplasie (Inzidenz 8/100 000). Diese Zahlen liegen unter der erwarteten Prävalenz von 4,9\% [13]. Ein Überwiegen von Mädchen fand sich im BiKeR-Register nicht.

Mit 6 Fällen ergab sich eine Prävalenz für einen Morbus Perthes von 158/100 000 und somit 20-fach höher als erwartet mit 3-12/100 000 [13]. Ein Geschlechterunterschied war nicht festzustellen. Die Inzidenz war niedrig $(8 / 100000 ; n=1)$.

Die Prävalenz einer Osteochondrosis dissecans war mit $184 / 100000(n=7)$ höher als erwartet (20-30/100 000 Kindern) [14]. Die Inzidenz war 32/100 $000(n=4)$. Statistisch ist eine Osteochondrosis dissecans bei Patienten mit persOA mit 3 von 492 Patienten signifikant häufiger (OR 5,1 [1,1-22,7]; $p=0,05)$. In 2 Fällen trat eine Osteochondrosis dissecans während der Beobachtung auf.

Eine Skoliose war im BiKeR-Register ( $n=21$, Prävalenz 0,55\%) seltener als erwartet (5,2\%) [6]. Ein signifikanter Unterschied bezüglich der Geschlechter bestand nicht. Bei Patienten mit uJIA $(n=3 / 123)$ besteht signifikant häufiger eine Skoliose (18/3680 Patienten; OR 5,1[1,5-17,5]; $p=0,004)$. Während der Beobachtung wurde 1 neuer Fall dokumentiert (Inzidenz 8/100 000).

Verlässliche Prävalenzdaten für einen Bandscheibenvorfall bei Kindern und Jugendlichen liegen nicht vor. In BiKeR fand sich eine Prävalenz 105/100 $000(n=4)$ und eine Inzidenz von $8 / 100000$ Jahre $(n=1)$. Patienten mit ERA (3/599) waren häufiger betroffen, allerdings kann aufgrund des möglichen Achsenskelettbefalls bei dieser JIA-Kategorie mit häufigeren bildgebenden Untersuchungen nicht ausgeschlossen werden, dass diese hierdurch nur häufiger diagnostiziert werden.

Zur Prävalenz der Osteoporose im Kinder- und Jugendalter liegen keine verlässlichen Daten vor. Auch erfolgten keine routinemäßigen Osteodensitometrien. Die Prävalenz berichteter Fälle war 315/100 000 ( $n=12) .2$ erhielten neu die Diagnose einer Osteoporose (Inzidenz 16/100 000) unter Therapie mit Kortikosteroiden und jeweils Etanercept bzw. Tocilizumab.

Verlässliche Daten zur Prävalenz von regionalen und generalisierten Schmerzsyndromen bei Kindern und Jugendlichen fanden sich nicht. Bei Erwachsenen beträgt die Prävalenz etwa $3 \%$ [14]. Es fand sich eine Prävalenz in BiKeR von 789/100 $000(n=30)$ und eine Inzidenz von 104/100 000 ( $n=16)$. Zwischen den Geschlechtern gab es keinen Unterschied.

Verlässliche Daten zur Prävalenz einer chronischen nicht bakteriellen Osteitis (CNO) in der allgemeinen pädiatrischen Bevölkerung ließen sich nicht eruieren. In BiKeR fand sich eine Prävalenz von 158/100 $000(n=6)$. Patienten mit ERA waren signifikant häufiger betroffen $(n=3$; OR 5,4 [1,1-26,7]; $p=0,02)$. Ein Patient erhielt neu die Diagnose CNO (Inzidenz 8/100 000).

\section{Kardiovaskuläre Erkrankungen}

Bei 7 Patienten bestand eine Aortenklappeninsuffizienz und bei 10 eine Mitralklappeninsuffizienz (Prävalenz 447/100 000). Verlässliche Referenzwerte zum Vorkommen bei Kindern und Jugendlichen liegen nicht vor. Ein Vorhofseptumdefekt oder Ventrikelseptumdefekt fand sich bei 9 Kindern (Prävalenz 237/100 000).

Bei 25 Patienten war eine arterielle Hypertonie bekannt (Prävalenz 657/100 000). Die Prävalenz ist niedriger als erwartet (1-3\%)
[15]. Patienten mit einer sJIA hatten häufiger eine arterielle Hypertonie (8/253, OR 6,8 [2,9-15,9]; $p<0,001)$. Von 10 Patienten (Inzidenz 80/100 000), die im Beobachtungsverlauf eine arterielle Hypertonie entwickelten, wurden 5 mit Etanercept, 4 mit Adalimumab und 1 mit Methotrexat behandelt.

\section{Psychiatrische Erkrankungen}

Angstzustände und Panikattacken zeigten eine Prävalenz von $53 / 100000(n=2)$ und eine Inzidenz 40/100 000 Jahre $(n=5)$, deutlich niedriger als erwartet (9,5\%) [16]. Für Suizidversuche bzw. eine Depression fand sich eine Prävalenz von 158/100 000 (n=6). Demgegenüber steht eine deutlich höhere Prävalenz der Depression von 4-8\% im Kinder- und Jugendalter [17]. Zwischen den unterschiedlichen JIA-Kategorien oder den Geschlechtern gab es keine Unterschiede. Die Inzidenz einer Depression war 144/100 000 Jahre $(n=16)$. Dabei waren PsA-Patienten signifikant häufiger betroffen (4/287; OR 3,5 [1,2-10,8], $p=0,05)$.

In der pädiatrischen Bevölkerung besteht eine Prävalenz für Aufmerksamkeitsstörungen von 3 \% [5]. Mit 24 Patienten war die Prävalenz in BiKeR 631/100 000, bei 1 neuem Patienten war die Inzidenz 8/100000. Jungen waren häufiger betroffen (18/1256; OR 6,2 $[2,4-15,6] ; p<0,00001) .13$ dieser Jungen hatten eine ERA, und somit war die Aufmerksamkeitsstörung ebenso signifikant häufiger (OR 6,59 [2,3-18,4]; $p<0,00005)$.

\section{Hämatologische Erkrankungen}

Eine Anämie lag mit einer Prävalenz von 552/100 000 ( $n=21)$ vor und damit unterhalb von 2,4\% nach dem bundesweiten Kinder- und Jugendgesundheitssurvey von 2009. Die Inzidenz war 16/100 000. Initial war bei keinem Patienten eine Leukozytopenie bekannt. Im Beobachtungszeitraum war die Inzidenz 463/100 $000(n=58)$. Patienten mit sJIA waren häufiger betroffen (9/253; OR 3,4 [1,6-7,1]; $\mathrm{p}>0,001)$ ebenso Patienten mit RF${ }^{+}-\mathrm{PA}(8 / 227$; OR 3,3 [1,5-7,2]; $p=0,001)$. Leukozytopenien traten besonders häufig unter der Behandlung mit Tocilizumab (11/69; $16 \%$ der behandelten Patienten) sowie CAN $(2 / 12,17 \%)$ und ANA $(1 / 9 ; 11 \%)$ auf.

\section{Genetisch bedingte Erkrankungen}

Bei 3 Kindern war eine Thalassämie bekannt (79/100 000 Patienten). Verlässliche Prävalenzwerte bezüglich Thalassämie in Deutschland fanden sich nicht. Für das Familiäre Mittelmeerfieber fand sich eine Prävalenz von 237/100 $000(n=9)$. Betroffen waren insbesondere sJIA-Patienten (3/253; OR 7,1 [1,8-28,5]; $p=0,02$ ). Ein Turner-Syndrom fand sich mit einer Prävalenz von $53 / 100000(n=2)$ bei in der Bevölkerung vorliegenden Prävalenz von 50/100 000 [18]. Das Down-Syndrom zeigte eine Prävalenz von 184/100 $000(n=7)$, v. a. bei PsA (4/287; OR 16,6 [3,7-74,3]; $p=0,001)$ und bei Jungen $(56 / 1256 ; O R=5,1[1,0-26,3] ; p=0,04)$.

\section{Gastrointestinale Erkrankungen}

Bei 5 Patienten bestand eine Zöliakie. Die Prävalenz war mit 125/100 000 niedriger als erwartet mit 0,9\% [19]. Statistisch ist eine Zöliakie bei RF+-PA (2/225) signifikant häufiger (OR 10,6 [1,764]; $p=0,03$ ). Es wurden 2 inzidente Fälle dokumentiert (Inzidenz 16/100 000). Definitionsgemäß bestanden bei Einschluss keine chronisch entzündlichen Darmerkrankungen (CED), weil dies einem Ausschlusskriterium entspricht. Bei 32 Patienten wurde eine inziden- 
te CED dokumentiert (Inzidenz 152/100 000). Die Prävalenz von chronisch entzündlichen Darmerkrankungen bei Kindern und Jugendlichen in der allgemeinen pädiatrischen Population ist mit 5-11/100000 [20] niedriger. 18 Fälle traten während einer Therapie mit Etanercept auf, 8 Fälle unter Methotrexat ohne Biologika. Betroffen waren vor allem Patienten mit sJIA und uJIA. Für die Fruktosemalabsorption zeigte sich eine Prävalenz von 105/100000 ( $n=4)$ und eine Inzidenz von 16/100000 ( $n=2)$. Vergleichswerte fanden sich nicht. Eine Laktoseintoleranz zeigte eine Prävalenz von 263/100 000 $(n=10)$ und eine Inzidenz von 16/100000 ( $n=2)$. Die Prävalenz in der adulten Bevölkerung in Deutschland liegt bei 15-20\% [21].

\section{Neurologische Erkrankungen}

Aufgrund der Erfassung im BiKeR-Register mittels des MedDRA-Systems wurden unter Entwicklungsverzögerung, low-level-term developmental delay, folgende Begriffe kodiert: Entwicklungsrückstand $(n=5)$, psychosomatische Retardierung $(n=3)$, Lernbehinderung, syndromale Entwicklungsstörung, Sprachentwicklungs-, motorische und globale Retardierung sowie körperliche und geistige Retardierung. Die Prävalenz war 1367/100000 ( $n=15)$ und niedriger als erwartet (13\%). Für Krampfanfälle war die Prävalenz mit 473/100000 ( $n=18)$ niedriger als erwartet (3,6\%). Die Inzidenz war 56/100 000 ( $n=7)$. Die Prävalenz der Migräne bei Kindern und Jugendlichen ist mit 2,5\% hoch [5]. Bei keinem Patienten war bei Ersterhebung eine Migräne angegeben. Bei 14 Kindern wurde unter Beobachtung eine Migräne diagnostiziert (Inzidenz 192/100 000).
Patienten mit RF--PA waren signifikant häufiger betroffen (8/1144 OR 3, 1 (95\%-KI 1,1-8,9; $p=0,04)$.

\section{Otorhinologische Erkrankungen}

Mit 7 Patienten (184/100 000) war die Prävalenz für Hörstörungen in BiKeR der pädiatrischen Bevölkerung entsprechend (100300/100 000) [22].

\section{Urologische Erkrankungen}

Die Prävalenz für einen vesikourethralen Reflux war mit 105/100 000 $(n=4)$ niedriger als erwartet (2-10\%) [6]. Es gab keine neuen Fälle.

\section{Onkologische Erkrankungen}

1 Patient war vor Einschluss in die Beobachtung an einer malignen Neoplasie erkrankt, an einer ALL (Prävalenz 26/100 000). Während der Beobachtung wurden 3 Fälle eines Morbus Hodgkin, je 2 von ALL und NHL und je 1 anaplastisches Ependymom, Schilddrüsenkarzinom und Gonadoblastom dokumentiert ( $n=10$, Inzidenz 79,8/100 000). 1 Patient wurde mit Tocilizumab behandelt, 2 ausschließlich mit Methotrexat, 2 nur mit Etanercept, 1 mit Etanercept und Azathioprin. Alle anderen standen unter Kombinationstherapie mit Etanercept und Methotrexat. Im Mittel trat die Neoplasie 2,8 Jahre nach Therapieeinleitung auf. Patienten mit sJIA waren häufiger betroffen als Patienten der übrigen JIA-Kategorien (2/253; OR = 3,5 [0,7-16,7]; $p=0,08)$. In der Geschlechterverteilung bestand kein Unterschied. In 2014 wurden in Deutschland 


\begin{tabular}{|c|c|c|c|c|c|c|c|}
\hline & sJIA & RF--PA & $\mathrm{RF}^{+}-\mathrm{PA}$ & persOA & erwOA & ERA & PSA \\
\hline Adipositas & $5,9 \%$ & $4,1 \%$ & $4,4 \%$ & $3,7 \%$ & $3,2 \%$ & $4,7 \%$ & $5,2 \%$ \\
\hline Allergie & $1,2 \%$ & $1,0 \%$ & $1,3 \%$ & $0,8 \%$ & $1,9 \%$ & $3,2 \%$ & $1,4 \%$ \\
\hline Alopezie & $0,4 \%$ & $0,1 \%$ & & $0,2 \%$ & $0,3 \%$ & & $0,3 \%$ \\
\hline Anämie & $0,8 \%$ & $0,5 \%$ & $0,9 \%$ & $0,2 \%$ & $0,7 \%$ & $0,3 \%$ & $0,7 \%$ \\
\hline Arterielle Hypertonie & $3,2 \%$ & $0,7 \%$ & & & $0,6 \%$ & $0,3 \%$ & $0,3 \%$ \\
\hline Asthma & $0,4 \%$ & $1,3 \%$ & $0,4 \%$ & $1,0 \%$ & $1,8 \%$ & $3,0 \%$ & $3,8 \%$ \\
\hline Atopische Dermatitis & $0,8 \%$ & $1,2 \%$ & $1,3 \%$ & $0,6 \%$ & $1,6 \%$ & $1,8 \%$ & $1,0 \%$ \\
\hline Aufmerksamkeitsstörung & $0,4 \%$ & $0,3 \%$ & $0,9 \%$ & $0,2 \%$ & $0,1 \%$ & $2,2 \%$ & $1,0 \%$ \\
\hline Bandscheibenvorfall & & $0,1 \%$ & & & & $0,5 \%$ & \\
\hline Chronisch entzündliche Darmerkrankungen & & $0,3 \%$ & & $0,2 \%$ & $0,1 \%$ & $0,3 \%$ & $0,3 \%$ \\
\hline Chronische nicht bakterielle Osteitis & & $0,2 \%$ & & $0,2 \%$ & & $0,5 \%$ & \\
\hline Cushingoid & $5,1 \%$ & $0,2 \%$ & $0,4 \%$ & & & & \\
\hline Depression & & $0,2 \%$ & & & $0,3 \%$ & & \\
\hline Diabetes mellitus & $0,4 \%$ & $0,9 \%$ & $1,3 \%$ & & $0,3 \%$ & $0,5 \%$ & $0,7 \%$ \\
\hline Familiäres Mittelmeerfieber & $1,2 \%$ & $0,3 \%$ & & $0,2 \%$ & $0,1 \%$ & & \\
\hline Funktionelle Herzerkrankungen & & $0,3 \%$ & & & $0,9 \%$ & $0,8 \%$ & $0,7 \%$ \\
\hline Hashimoto-Thyreoiditis & $0,8 \%$ & $1,1 \%$ & $3,5 \%$ & $0,4 \%$ & $0,6 \%$ & $1,0 \%$ & $4,2 \%$ \\
\hline Hörstörung & & $0,3 \%$ & & & & $0,2 \%$ & $0,7 \%$ \\
\hline Hüftdysplasie & $0,8 \%$ & $0,6 \%$ & & $0,2 \%$ & $0,3 \%$ & $0,5 \%$ & \\
\hline Hypogammaglobulinämie & & & & & $0,4 \%$ & & \\
\hline IgA-Mangel & & $0,4 \%$ & $0,4 \%$ & $0,2 \%$ & $1,0 \%$ & $0,3 \%$ & \\
\hline Kleinwuchs & $16,6 \%$ & $4,0 \%$ & $7,5 \%$ & $1,8 \%$ & $4,0 \%$ & $3,5 \%$ & $4,9 \%$ \\
\hline Krampfanfälle & $0,4 \%$ & $0,3 \%$ & $1,3 \%$ & & $0,6 \%$ & $0,8 \%$ & $0,3 \%$ \\
\hline Laktoseintoleranz & & $0,4 \%$ & & $0,6 \%$ & & $0,2 \%$ & $0,3 \%$ \\
\hline Makrophagenaktivierungssyndrom & $3,6 \%$ & $0,1 \%$ & & & & & \\
\hline Maligne Neoplasien & & & & & & $0,8 \%$ & \\
\hline Morbus Perthes & $0,4 \%$ & & & $0,2 \%$ & $0,3 \%$ & $0,2 \%$ & $0,3 \%$ \\
\hline Osteochondritis dissecans & & $0,1 \%$ & & $0,6 \%$ & $0,3 \%$ & $0,2 \%$ & \\
\hline Osteoporose & $0,8 \%$ & $0,3 \%$ & $1,3 \%$ & & $0,3 \%$ & & \\
\hline Psoriasis & $0,4 \%$ & $0,3 \%$ & & & $0,1 \%$ & $0,5 \%$ & 10,5 \\
\hline Raynaud-Syndrom & & $0,3 \%$ & $0,4 \%$ & & & & $0,3 \%$ \\
\hline Reifungsverzögerung & & $0,6 \%$ & $0,4 \%$ & $0,0 \%$ & $0,6 \%$ & $0,2 \%$ & $0,7 \%$ \\
\hline Schmerzsyndrome & & $1,0 \%$ & $0,4 \%$ & $1,0 \%$ & $0,6 \%$ & $1,2 \%$ & $0,3 \%$ \\
\hline Skoliose & & $0,3 \%$ & & $0,4 \%$ & $0,4 \%$ & $0,8 \%$ & $1,4 \%$ \\
\hline Strukturelle Herzfehler & & $0,3 \%$ & $0,4 \%$ & $0,2 \%$ & $0,3 \%$ & $0,3 \%$ & \\
\hline Trisomie 21 & & $0,3 \%$ & & & & & $1,4 \%$ \\
\hline Urtikaria & $0,8 \%$ & $0,2 \%$ & & & & & \\
\hline Uveitis & $0,8 \%$ & $5,8 \%$ & $1,3 \%$ & $17,3 \%$ & $20,8 \%$ & $6,3 \%$ & $5,2 \%$ \\
\hline Vaskulitis & $0,8 \%$ & $0,1 \%$ & $0,4 \%$ & & & & \\
\hline Vesikoureteraler Reflux & & & & & $0,4 \%$ & $0,2 \%$ & \\
\hline Vitiligo & & $0,3 \%$ & $0,4 \%$ & $0,2 \%$ & & $0,2 \%$ & $0,3 \%$ \\
\hline
\end{tabular}

grün =Anteil der Patienten mit Komorbidität $>0,1 \%$ und $<1-5$; gelb = Anteil der Patienten mit Komorbidität $\geq 1 \%, \leq 5 \%$; rot $=$ Anteil der Patienten mit Komorbidität

Abb. 2 Vorkommen von Komorbiditäten nach JIA-Kategorie. 
- Tab. 4 Antirheumatische Therapie und inzidente Komorbiditäten.

\begin{tabular}{|l|l|l|l|}
\hline Antirheumatische Therapie & Komorbiditäten gesamt & Anzahl Pat. mit Komorbidität & Rate Pat. mit Komorbidität \\
\hline Methotrexat $(n=3067)$ & 263 & 97 & $3 / 100$ \\
\hline Etanercept $(n=1937)$ & 343 & 236 & $12 / 100$ \\
\hline Adalimumab $(n=269)$ & 82 & 72 & $27 / 100$ \\
\hline Tocilizumab $(n=69)$ & 39 & 36 & $54 / 100$ \\
\hline Azathioprin $(n=90)$ & 4 & 4 & $4 / 100$ \\
\hline Leflunomid $(n=55)$ & 3 & 3 & $6 / 100$ \\
\hline Cyclosporin A $(n=58)$ & 4 & 4 & $7 / 10$ \\
\hline
\end{tabular}

1657 Malignome bei 10,5 × $10^{6}$ Kindern < 15 Jahre beobachtet, entsprechend einer Inzidenz von 15,8/100 000 [23]. Damit ist die Inzidenz maligner Erkrankungen bei der JIA signifikant erhöht (RR 5,1 $[2,4-9,4] ; p<0,001)$.

\section{Geschlechterspezifische Unterschiede}

Jungen mit JIA hatten signifikant häufiger eine Aufmerksamkeitsstörung (OR 6,2 [2,4-15,6]), ein Asthma (OR 1,9 [1,1-3,1]), eine Trisomie 21 (OR 5,1 [1-26,3]) oder eine CED (OR 3,3 [1,1-10]). Mädchen hatten signifikant häufiger eine Hashimoto-Thyreoiditis (OR 3,6 [1,3-10,3]) und grenzwertig signifikant häufiger ein MAS (OR 3, $1[0,9-10,6])$.

\section{Unterschiede zwischen den JIA-Kategorien}

Patienten mit sJIA sind häufiger (16,6\%) kleinwüchsig oder adipös, hatten ein Cushingoid, eine arterielle Hypertonie oder ein MAS, seltener eine Uveitis ( $\triangleright$ Tab. 1). Bei RF'-PA bestanden häufiger Kleinwuchs, Hashimoto-Thyreoiditis, Diabetes mellitus oder Osteoporose, seltener eine Uveitis oder ein Asthma. Die führenden Komorbiditäten bei RF--PA sind Uveitis, Kleinwuchs, gefolgt von Asthma, atopischer Dermatitis, Schmerzsyndromen, Allergien und Hashimoto-Thyreoiditis. Bei der persOA und erwOA ist eine Uveitis mit 17-21\% wesentlich häufiger, Patienten sind seltener adipös oder kleinwüchsig. Patienten mit ERA haben häufiger Allergien, Asthma und atopische Dermatitis oder Aufmerksamkeitsstörung. Wie zu erwarten kommt Psoriasis bei der PsA weitaus am häufigsten vor. Häufiger sind zudem Skoliose, Asthma oder Hashimoto-Thyreoiditis. Unterschiede in der Inzidenz von Komorbiditäten sind in $>$ Abb. 2 farblich verdeutlicht.

\section{Antirheumatische Therapie}

Den Einfluss der antirheumatischen Therapie auf die Prävalenz und Inzidenz von Komorbiditäten zeigt $>$ Tab. 4. Methotrexat und Etanercept werden wesentlich häufiger verwendet als die übrigen DMARDs mit niedriger Rate von neu aufgetretenen Komorbiditäten als unter Therapie mit Adalimumab und Tocilizumab. Sehr selten kamen Abatacept, Infliximab, Canakinumab, Anakinra und Golimumab zum Einsatz, weshalb diese nicht ausgewertet wurden.

\section{Diskussion}

Grundlage für die Untersuchung von Komorbiditäten bei JIA ist das BiKeR-Register. Für die Aussagekraft der Untersuchung zur Prävalenz und Inzidenz der Komorbiditäten bei JIA stellt die außerordentliche Größe von über 4000 Patienten sowie die lange Beobachtung der Patienten einen enormen Vorteil dar.

Bezüglich der Komorbiditäten bei JIA sind nur wenige Daten verfügbar. Eine Umfrage selbstberichteter Komorbiditäten bei erwachsenen JIA-Patienten ergab bei $62 \%$ mindestens eine Komorbidität [3]. Führend war die Uveitis mit 17,7\%, gefolgt von Migräne und atopischen Erkrankungen. Kardiovaskuläre Erkrankungen waren nicht häufiger als in der Referenzgruppe. Nur Patienten mit sJIA zeigten eine erhöhte Rate kardiovaskulärer Erkrankungen (40,6\%).

Eine Studie zum Langzeit-Outcome bei 256 JIA-Patienten von 1978 und 1988 bezüglich Veränderungen der Körperfunktion und -struktur, Mortalität, Gelenkveränderungen, Krankheitsaktivität zeigte etwa bei der Hälfte der Patienten nach im Mittel 16,5 Krankheitsjahren noch eine aktive Erkrankung. $1 / 3$ der Patienten stufte sich als funktionseingeschränkt ein. Begleiterkrankungen waren Uveitis bei $1 / 3$, kardiale Beteiligung (Perikarditis und Myokarditis) bei $5 \%$, Kleinwuchs bei 1,4\% der Patienten, lokale Wachstumsstörungen bei $1 \frac{1}{4}$, Die Mortalität war nicht erhöht [24].

Die Uveitis ist mit $12 \%$ die häufigste extraartikuläre Manifestationsform der JIA. Die Prävalenz unterscheidet sich je nach JIAKategorie. Vor allem Patienten mit oligoartikulärer JIA, positivem ANA-Titer und jungem Alter bei Diagnosestellung haben ein erhöhtes Uveitisrisiko [25]. Komplikationen der Uveitis, Katarakt, Glaukom oder Makulaödem können zum Visusverlust führen.

Ein gehäuftes Vorkommen von Wachstumsverzögerungen bei Kindern mit Diabetes mellitus Typ I, juveniler chronischer Arthritis und Asthma wurde bereits beschrieben. Mögliche Ursachen sind Kortikosteroidtherapie, Mangelernährung, verminderte körperliche Aktivität und erhöhte Spiegel proinflammatorischer Zytokine. Unter langfristiger Steroidtherapie war eine signifikante Assoziation zu einer reduzierten Körpergröße im Erwachsenenalter festgestellt worden. Nach Remission der JIA und vor allem nach Beendigung der Steroidtherapie ist ein Aufholwachstum möglich [26].

Eine erhöhte Prävalenz von Autoimmunthyreoiditis (11,9\% der Patienten; $p<0,0001)$, subklinische Hypothyreose $(9,3 \%$ der Patienten; $p<0,01)$ und Zöliakie $(6,6 \%$ der Patienten; $p<0,005)$ bei Kindern mit JIA ist vorbeschrieben [27]. 
In einer skandinavischen Studie bestanden bei 3,1\% der Kinder mit JIA weitere Autoimmunerkrankungen, Diabetes mellitus Typ I, Zöliakie oder Hypothyreose mit 5 fach erhöhtem Risiko [28]. Das Risiko, an einem Diabetes Typ I zu erkranken, war 2fach erhöht. Diskutiert werden noch höhere Raten aufgrund verzögerter Diagnose oder Auftreten erst im Erwachsenenalter. Die Prävalenz des Diabetes mellitus Typ I ist bei der hier untersuchten Population deutlich höher als in der allgemeinen pädiatrischen Bevölkerung (0,6\% versus $0,14 \%-0,28 \%)$. Mehrere Untersuchungen weisen auf ein häufigeres Vorliegen eines Diabetes mellitus Typ I hin. So liegt die Prävalenz von Diabetes mellitus Typ I (DM1) bei Kindern mit JIA bei $0,5 \%[28,29] .63 \%$ der Patienten entwickeln den DM1 vor der JIA. Die meisten Patienten, bei denen der DM1 nach der JIA auftritt, haben vorher noch keine DMARDs erhalten, zudem fand sich auch ein früheres Auftreten des Diabetes als bei Patienten ohne JIA [30].

Bereits publizierte Daten des BiKeR-Registers beschreiben eine erhöhte Inzidenz an chronisch entzündlichen Darmerkrankungen (CED). Patienten, welche mit Methotrexat behandelt wurden, zeigten eine signifikant niedrige Inzidenz, eine Monotherapie mit Etanercept war mit einer erhöhten CED-Inzidenz assoziiert [31].

Das Makrophagenaktierungssyndrom (MAS) ist eine typische Komplikation der sJIA. Eine Expansion von Makrophagen bei 30\% der sJIA-Patienten ist bekannt. Dies ist als mögliches Frühstadium eines MAS zu bewerten [8]. Es ist als Komplikation von weiteren Autoimmunerkrankungen bekannt und charakterisiert durch eine exzessive Aktivierung von Makrophagen im Rahmen einer Fehlregulation des Immunsystems. Die frühzeitige Diagnose ist entscheidend, aufgrund des unspezifischen, septischen Krankheitsbildes jedoch schwierig [32]. Bei ca. 1,5-3,7\% der Patienten mit JIA tritt im Verlauf ein MAS auf $[32,33]$. Bei sJIA sind deutlich höhere Raten von 7-10\% beschrieben [8]. Die in BiKeR gefundene Prävalenz bei JIA von $0,26 \%$ und Inzidenzrate von $0,1 \%$, bzw, bei s sIA eine Inzidenz von $4,7 \%$ entspricht der Literatur.

Insgesamt lag die Prävalenz atopischer Erkrankungen und von Asthma niedriger als erwartet. Dies entspricht bereits publizierten Daten [34]. Als ursächlich wurden die durch Th1 bzw. Th2 vermittelten Zytokinmuster bewertet. Eine Th1-vermittelte Immunantwort wird durch die Anwesenheit von Th2-vermittelten Zytokinen inhibiert [35].

Die Prävalenz der Adipositas ist mit 4,4\% niedriger als erwartet (6,3\%, Stand 2010). Dies erstaunt, da aufgrund der durch die JIA bedingten Bewegungseinschränkung eher eine erhöhte Prävalenz der Adipositas erwartet wurde.

Die erhöhte Prävalenz eines Morbus Perthes oder einer Osteochondritis dissecans bei Patienten in BiKeR kann durch die von chronischer Entzündung getriggerten Ischämie oder aufgrund vermehrter Aufmerksamkeit und häufiger Bildgebung bedingt sein. Relevante Studien zu einem Zusammenhang zwischen JIA und M. Perthes gibt es bisher nicht. Eine Osteochondritis dissecans ist auch bei Patienten mit JIA in Abwesenheit von Entzündungszeichen vorbeschrieben. Nicht auszuschließen ist ein ungünstiger Effekt intraartikulärer Steroidapplikationen [36].

Osteoporose ist bei Patienten mit rheumatischen Erkrankungen multifaktoriell durch inflammatorische Zytokine, Glukokortikoidtherapie, verminderte körperliche Aktivität, schmerzhafte Gelenke und Muskelschwäche bedingt [37]. Bezüglich der Prävalenz und
Inzidenz bei der JIA werden jedoch keine Daten gefunden. 0,3\% der Patienten mit JIA haben eine Osteoporose.

Die CNO ist mit einer Prävalenz von 0,2\% eine Seltenheit, vor allem weibliche Patienten mit ERA sind betroffen. Eine CNO ist mit verschiedenen Erkrankungen assoziiert, so mit einer chronisch entzündlichen Darmerkrankung, pustulösen und psoriatiformen Hauterkrankungen und dem HLA-B27.

Zu kardiovaskulären Erkrankungen bei JIA finden sich wesentlich weniger Untersuchungen als zu kardiovaskulären Erkrankungen bei der rheumatoiden Arthritis (RA) des Erwachsenen. So ist bei Patienten mit RA das Risiko für eine kardiovaskuläre Erkrankung (inklusive Myokardinfarkt, zerebrovaskuläre Ereignisse und Herzinsuffizienz) um 48 \% erhöht, verglichen mit der Allgemeinbevölkerung [38]. Eine verdoppelte Rate an Myokardinfarkt und Schlaganfall ist bei Patienten mit RA bekannt [39]. Dort wird auch dargestellt, dass das relative Risiko für kardiovaskuläre Ereignisse bei Patienten mit RA für junge Patienten und für Patienten mit vorangegangenen kardiovaskulären Ereignissen am höchsten ist.

Die Prävalenz der arteriellen Hypertonie bei pädiatrischen JIA-Patienten liegt bei $0,7 \%$ und hiermit unter der Prävalenz von $10-30 \%$, die für Kinder und Jugendliche bekannt ist. Bezieht man jedoch zu den prävalenten und inzidenten Fällen an arterieller Hypertonie auch noch diejenigen Patienten mit ein, bei denen keine arterielle Hypertonie als Diagnose in BiKeR dokumentiert ist, die aber unter antihypertensiver Therapie stehen, so sind 1,7\% der Patienten betroffen. Zu erwarten wäre auch eher eine erhöhte Prävalenz an Hypertonie, die unter anderem als Nebenwirkung einer Steroidtherapie auftritt. Hierzu korrespondiert das gehäufte Vorkommen einer arteriellen Hypertonie bei sJIA. Bei Kindern mit JIA wurde, im Vergleich zu gesunden Kindern, eine höhere Rate an Angststörungen, Depression, regelbrechendem und aggressivem Verhalten gefunden [40]. Die Prävalenz von Schmerzsyndromen bei Kindern mit JIA ist deutlich niedriger als die Prävalenz bei RA-Patienten von 13,4\% [41].

Auch war die Adipositas-Prävalenz bei JIA mit der der allgemeinen pädiatrischen Population vergleichbar und nahm über die Jahre 2003-2012 ab. Hierfür wurde vermehrte körperliche Aktivität sowie Reduktion einer hochdosierten Glukokortikoidtherapie verantwortlich gemacht [42].

Ein Cushingoid (iatrogenes Cushing-Syndrom) bei JIA wurde vor allem im Zusammenhang mit intraartikulären Triamcinolonaceton-Injektionen beschrieben $[43,44]$.

Studien zu Malignomen bei Patienten mit JIA zeigen eine erhöhte Malignomrate ohne signifikante Assoziation zur Therapie [45]. Bei ostasiatischen Kindern mit JIA fanden Kok et al. ein 3fach erhöhtes Risiko für Malignome, welches weder durch eine Therapie mit Methotrexat noch mit TNF-Inhibitoren erhöht wurde [46]. Für Europa fand sich ein 3fach erhöhtes Risiko für Malignome bei JIA-Patienten ohne eine Biologikatherapie [47].

Die wesentliche Limitierung der vorgestellten Analyse ist die mangelnde Möglichkeit der Verifizierung der Korrektheit der Nebendiagnosen. Zudem ist anzunehmen, dass nicht alle Komorbiditäten berichtet und dokumentiert wurden, obschon durch Monitoringvisiten in den größeren Zentren überprüft. Des Weiteren ist das Patientenkollektiv nicht repräsentativ, sondern eine Negativauslese. Die hier untersuchten Daten sind dem BiKeR-Register entnommen, in welchem nur JIA-Patienten verfolgt werden, die 
eine Therapie mit Methotrexat oder mit Biologika erhielten, leichtere Fälle also ausgeschlossen waren.

Interessenkonflikt

Das deutsche Register wird durch eine unabhängige Förderung seitens Pfizer (Deutschland), AbbVie (Deutschland), MSD (Deutschland) und Roche (Deutschland) unterstützt. AbbVie, Pfizer, MSD und Roche hatten keinerlei Einfluss auf das Studiendesign oder die Sammlung, Analyse oder Interpretation der Daten, das Verfassen des Manuskripts oder die Entscheidung, das Manuskript zur Veröffentlichung einzureichen. Die Veröffentlichung dieses Artikels war nicht von der Genehmigung durch die Studiensponsoren abhängig.

G.H.: Unterstützung wissenschaftlicher Projekte durch AbbVie, Chugai, MSD, Novartis, Pfizer, Roche und Honorare für Vorträge von AbbVie, Bayer, Chugai, Novartis, Pfizer, Roche und Sobi R.N.: keine

\section{Einhaltung ethischer Richtlinien}

Dem BiKeR-Register liegt eine Befürwortung der zentralen Ethikkommission der Ärztekammer Nordrhein, Düsseldorf, vor. Die schriftliche Zustimmung der Patienten und Eltern wurde eingeholt und wiederholt, wenn der Patient erwachsen wurde. Es wurden nur pseudonymisierte Daten gesammelt.

Danksagung

Die Autoren danken zahlreichen Kinderrheumatologinnen und -rheumatologen für die Zuarbeit und ebenso den Patienten und Eltern für die anhaltende Unterstützung.

\section{Literatur}

[1] Petty RE, Spouthwood TR, Manners P et al. International League of Associations for Rheumatology: International League of Associations for Rheumatology classification of juvenile idiopathic arthritis: second revision, Edmonton 2001.J Rheumatol 2004; 31(2): 390392

[2] Flatø B, Aasland A, Vinje O, Førre O. Outcome and predictive factors in juvenile rheumatoid arthritis and juvenile spondyloarthropathy. J Rheumatol 1998; 25(2): 366-375

[3] Raab A, Sengler C, Niewerth M et al. Comorbidity profiles among adult patients with juvenile idiopathic arthritis: results of a biologic register. Clin Exp Rheumatol 2013; 31(5): 796-802

[4] Neuhauser H, Schienkiewitz A, Schaffrath Rosario A et al. Referenzperzentile für anthropometrische Maßzahlen und Blutdruck aus der Studie zur Gesundheit von Kindern und Jugendlichen in Deutschland (KiGGS) in Beiträge zur Gesundheitsberichterstattung des Bundes 2. erweiterte Auflage, RKI-Hausdruckerei, Berlin

[5] Mayatepek E. Pädiatrie. München: Elsevier (Urban \& Fischer), 2007

[6] Kamtsiuris P, Atzpodien K, Ellert U et al. Prävalenz von somatischen Erkrankungen bei Kindern und Jugendlichen in Deutschland. Robert Koch-Institut, Bundesgesundheitsblatt 2007; 50: 686-700

[7] Behrens EM, Beukelman T, Paessler M, Cron RQ. Occult macrophage activation syndrome in patients with systemic juvenile idiopathic arthritis. J Rheumatol 2007; 34: 113 
[8] Grom AA, Ilowite NT, Pascual V et al.; Paediatric Rheumatology International Trials Organisation and the Pediatric Rheumatology Collaborative Study Group. Rate and Clinical Presentation of Macrophage Activation Syndrome in Patients With Systemic Juvenile Idiopathic Arthritis Treated With Canakinumab. Arthritis Rheumatol 2016; 68(1): 218-228

[9] Kurth BM, Schaffrath Rosario A. Übergewicht und Adipositas bei Kindern und Jugendlichen in Deutschland. Robert Koch-Institut, Bundesgesundheitsblatt 2010; 53: 643-652

[10] S3-Leitlinie Urtikaria. http://dgaki.de/wp-content/uploads/2010/05/ Leitlinie-Urtikaria_Teil1_AJ_052011_20_249_58.pdf

[11] Krüger C, Schallreuter KU. A review of the worldwide prevalence of vitiligo in children/adolescents and adults. International Journal of Dermatology 2012; 51: 1206-1212

[12] Schäfer I, Rustenbach S], Radtke M et al. Epidemiologie der Psoriasis in Deutschland - Auswertung von Sekundärdaten einer gesetzlichen Krankenversicherung. Gesundheitswesen 2011; 73(5): 308-313

[13] Partenheimer A, Scheler-Hofmann M, Lange J et al. Populationsbasierte Studie zu Prädispositionsfaktoren und Häufigkeit der Hüftgelenksdysplasie. UltraschallinMed 2006; 27: 364-367

[14] Tscherne H. Unfallchirurgie im Kindesalter, Band 2. Berlin, Heidelberg, New York: Springer,2006

[15] Jahnsen K, Strube H, Starker A. Hypertonie. Gesundheitsberichterstattung des Bundes, Heft 43. Robert Koch Institut, Statistisches Bundesamt, Berlin 2008

[16] Federer M, Margraf J, Schneider S. Leiden schon Achtjährige an Panik? Zeitschrift für Kinder- und Jugendpsychiatrie und Psychotherapie 2000; 28: 205-214

[17] Leitlinie Suizidalität im Kindes- und Jugendalter: https://www.awmf. org/uploads/tx_szleitlinien/028-031I_S2k_Suizidalitaet_KiJu_201607_01.pdf

[18] Stochholm K, Juul S, Juel K et al. Prevalence, Incidence, Diagnostic Delay, and Mortality in Turner Syndrome, The Journal of Clinical Endocrinology \& Metabolism 2006; 91(10): 3897-3902

[19] Laass MW, Schmitz R, Uhlig et al. Zöliakieprävalenz bei Kindern und Jugendlichen in Deutschland. Dtsch Arztebl Int 2015; 112(33-34): 553-560

[20] Däbritz ], Gerner P, Enninger A et al. Chronisch-entzündliche Darmerkrankungen bei Kindern und Jugendlichen. Dtsch Arztebl Int 2017; 114(19): 331-338

[21] Terjung B, Lammert F. Laktoseintoleranz: Neue Aspekte eines alten Problems. Dtsch Med Wochenschr 2007; 132: 271-275

[22] Finckh-Krämer U, Gross M, Bartsch M et al. Hörscreening von Neugeborenen mit Risikofaktoren. HNO 2000; 48: 215-220

[23] Kaatsch P. Jahresbericht Kinderkrebsregister 2015. http://www.kinderkrebsregister.de (20 January 2016, date last accessed)

[24] Minden K, Niewerth M, Listing J et al. Long- term outcome in patients with juvenile idiopathic arthritis. Arthritis \& Rheumatism 2002; 46: 2392-2401

[25] Foeldvari I, Walscheid K, Heiligenhaus A. Uveitis bei juveniler idiopathischer Arthrtis. Z Rheumatol 2017; 76: 664

[26] Giannini C, Mohn A, Chiarelli F. Growth Abnormalities in Children with Type 1 Diabetes, Juvenile Chronic Arthritis, and Asthma. International Journal of Endocrinology 2014; 2014: 265954

[27] Stagi S, Giani T, Simonini G, Falcini F. Thyroid function, autoimmune thyroiditis and coeliac disease in juvenile idiopathic arthritis. Rheumatology (Oxford) 2005; 44(4): 517-520

[28] Pohjankoski $\mathrm{H}$, Kautiainen $\mathrm{H}$, Kotaniemi K et al. Autoimmune diseases in children with juvenile idiopathic arthritis. Scand J Rheumatol 2010; 39(5): 435-436
[29] Schenck S, Rosenbauer J, Niewerth M et al. Comorbidity of Type 1 Diabetes Mellitus in Patients with Juvenile Idiopathic Arthritis. J Pediatr 2018; 192: 196-203

[30] Chandrakasan S, Prahalad S. Revisiting Type 1 Diabetes as a Comorbidity in Patients with Juvenile Idiopathic Arthritis. J Pediatr 2018; 192: 6-7

[31] Barthel D, Ganser G, Kuester RM et al. Inflammatory Bowel Disease in Juvenile Idiopathic Arthritis Patients Treated with Biologics. J Rheumatol 2015; 42(11): 2160-2165

[32] An Q, Jin MW, An X] et al. Macrophage activation syndrome as a complication of juvenile rheumatoid arthritis. Eur Rev Med Pharmacol Sci 2017; 21(19): 4322-4326

[33] Silva CA, Silva CH, Robazzi TC et al. Macrophage activation syndrome associated with systemic juvenile idiopathic arthritis. J Pediatr (Rio J) 2004; 80(6): 517-522

[34] Hilliquin P, Allanore Y, Coste J et al. Reduced incidence and prevalence of atopy in rheumatoid arthritis. Results of a case-control study; Rheumatology (Oxford) 2000; 39(9): 1020-1026

[35] Rudwaleit M, Andermann B, Alten R et al. Atopic disorders in ankylosing spondylitis and rheumatoid arthritis. Annals of the Rheumatic Diseases 2002; 61: 968-974

[36] Kröger L, Piippo-Savolainen E, Tyrväinen E et al. Osteochondral lesions in children with juvenile idiopathic arthritis. Pediatr Rheumatol Online J 2013; 11(1): 18

[37] Maruotti N, Corrado A, Cantatore FP. Osteoporosis and rheumatic diseases. Reumatismo 2014; 66(2): 125-135

[38] Avina-Zubieta JA, Thomas J, Sadatsafavi M et al. Risk of incident cardiovascular events in patients with rheumatoid arthritis: a meta-analysis of observational studies. Annals of the Rheumatic Diseases 2012; 71: 1524-1529

[39] Solomon DH, Goodson N], Katz JN et al. Patterns of cardiovascular risk in rheumatoid arthritis. Annals of the Rheumatic Diseases 2006; 65: $1608-1612$

[40] Memari AH, Chamanara E, Ziaee V et al. Behavioral Problems in Juvenile Idiopathic Arthritis: A Controlled Study to Examine the Risk of Psychopathology in a Chronic Pediatric Disorder. Int J Chronic Dis 2016; 2016: 5726236

[41] Ranzolin A, Brenol JC, Bredemeier M et al. Association of concomitant fibromyalgia with worse Disease Activity Score in 28 joints, Health Assessment Questionnaire, and Short Form 36 scores in patients with rheumatoid arthritis. Arthritis Rheum 2009; 61: 794-800

[42] Schenck S, Niewerth M, Sengler C et al. Prevalence of overweight in children and adolescents with juvenile idiopathic arthritis. Scand J Rheumatol 2015; 44(4): 288-295

[43] Gondwe JS, Davidson JE, Deeley S et al. Secondary Cushing's syndrome in children with juvenile idiopathic arthritis following intra-articular triamcinolone acetonide administration. Rheumatology (Oxford) 2005; 44(11): $1457-1458$

[44] Kumar S, Singh RJ, Reed AM, Lteif AN. Cushing's syndrome after intra-articular and intradermal administration of triamcinolone acetonide in three pediatric patients. Pediatrics 2004; 113(6): 1820-1824

[45] Beukelman T, Haynes K, Curtis JR et al. Safety Assessment of Biological Therapeutics Collaboration. Rates of malignancy associated with juvenile idiopathic arthritis and its treatment. Arthritis Rheum 2012; 64(4): 1263-1271. doi: 10.1002/art.34348. Epub 2012 Feb 10

[46] Kok VC, Horng JT, Huang JL et al. Population-based cohort study on the risk of malignancy in East Asian children with juvenile idiopathic arthritis. BMC Cancer 2014; 14: 634

[47] Nordstrom BL, Mines D, Gu Y et al. Risk of malignancy in children with juvenile idiopathic arthritis not treated with biologic agents. Arthritis Care Res (Hoboken) 2012; 64(9): 1357-1364 